\title{
Towards Face Recognition Using Eigenface
}

\author{
Md. Al-Amin Bhuiyan \\ Department of Computer Engineering \\ King Faisal University \\ Hofuf, Al-Ahsa 31982, Saudi Arabia
}

\begin{abstract}
This paper presents a face recognition system employing eigenface-based approach. The principal objective of this research is to extract feature vectors from images and to reduce the dimension of information. The method is implemented on frontal view facial images of persons to explore a twodimensional representation of facial images. The system is organized with RMS (Root Mean Square) contrast scaling technique employed for pre-processing the images to adjust with poor lighting conditions. Experiments have been conducted using Carnegie Mellon University database of human faces and University of Essex Computer Vision Research Projects dataset. Experimental results indicate that the proposed eigenface-based approach can classify the faces with accuracy more than $80 \%$ in all cases.
\end{abstract}

Keywords-Eigenvector; Eigenface; RMS Contrast Scaling; Face Recognition

\section{INTRODUCTION}

Face recognition has attained an overwhelming popularity in image processing, computer vision, pattern recognition, and so on. The major application areas for face recognition include human-machine interface, surveillance systems, credit card verification, security systems, financial transactions, criminal identification, and so on. Although human beings are excellent in recognizing faces, it is not evident how faces are being associated with human memory. Since faces represent complex and multi-dimensional visual information, developing a computational model for face recognition is, therefore, quiet a challenging job.

A number of approaches have been cited in literature on face recognition. Sirovich and Kirby [1] introduced the concept of eigenface for recognition and Turk and Pentland [2] have applied the approach in face classification. They derived the eigenvectors from the covariance matrix of the probability distribution over the high-dimensional vector space of facial images. Wiskott [3], et al. established a Gabor wavelet-based elastic bunch graph matching system to label and identify facial images where face is characterized as a graph. Each node of graphs contain a list of parameters known as jets. However, both dynamic link architecture and elastic bunch graph matching methods need huge volumes of computational cost due to point-to-point matching or graph matching and these are not appropriate for real-time requirements. Lades, et al. [4] represented facial images by Gabor filters and developed a face recognition system employing dynamic link topology. Shan, et al. [5] proposed an enhanced fisher approach employing AdaBoost architecture for face recognition. Zhang, et al. [5] developed a face recognition system employing histogram of Gabor feature arrangement.
Liu and Wechsler [6] established a Gabor filter based identification method for dimensional reduction employing Fisher linear discriminate model. Kirby [8] and Terzopoulos, et al. [9] analysed the facial images on the bsis of facial characteristics. $\mathrm{Wu}$ [10] and Manjunath [11], et al. performed face recognition with feature vectors extracted from profile silhouettes. Kerin, et al. [12] have prposed a face recognition system employing neural network in tendem with selforganizing feature map. Nakamura, et al. [13] utilized isodensity maps to establish a face recognition system. Yullie, et al. [14] extracted feature vectors from the facial components like eyes, nose, mouth, and and employed deformable templates to the extraction of contours for facial images and outlined a face recognition procedure. Thakur et al. [15] proposed a face recognition system using principal component analysis (PCA) and radial basis function (RBF) neural network. The RBF network was designed by considering intra-class discriminating characteristics of the training images.

This paper explores Principle Component Analysis (PCA), which is applied on a characteristic dataset of facial images. The method is based on projecting images into a feature space that amounts the significant discrepancies among known facial images. These momentous features named as "Eigenfaces" are the principal components of the set of training facial images. Facial objects are then identified employing a nearestneighbor classifier.

The rest of the paper is organized as follows. Section II highlights image pre-processing. Section III describes Principle Component Analysis. The process of face detection is addressed in section IV. Face image normalization is described in Section V. Section VI illustrates the algorithms for face recognition. The experimental result has been presented in Section VII. Section VIII draws the overall conclusions of the research.

\section{IMAGE PRE-PROCESSING}

The original images in the face databases contain both color and grey scale images with different illumination conditions. Therefore, to make the images contrast invariant in terms of bright or dark environments, these are processed with same RMS contrast equalization. The RMS contrast metric is given by $[16,17]$ :

$$
C_{r, r m s}=\left[\frac{1}{P Q} \sum_{p=0}^{P-1} \underset{q=0}{Q-1}(r(p, q)-\bar{r})^{2}\right]^{1 / 2}
$$




$$
\begin{aligned}
& C_{g, r m s}=\left[\frac{1}{P Q} \underset{p=0}{P} \underset{q=0}{\sum-1}(g(p, q)-\bar{g})^{2}\right]^{1 / 2} \\
& C_{b, r m s}=\left[\frac{1}{P Q} \sum_{p=0}^{P-1} \sum_{q=0}^{Q-1}(b(p, q)-\bar{b})^{2}\right]^{1 / 2}
\end{aligned}
$$

where $r(p, q), g(p, q), b(p, q)$ denote the illumination owing to red, green, and blue color constituents, respectively, and $\bar{r}$, blue color components. All facial images are retained the same lighting conditions applying the following equation:

$$
\mathbf{f}_{r}=\alpha_{r} \mathbf{C}_{r}+\beta_{r}, \mathbf{f}_{g}=\alpha_{g} \mathbf{C}_{g}+\beta_{g}, \mathbf{f}_{b}=\alpha_{b} \mathbf{C}_{b}+\beta_{b}
$$

where $\alpha_{r}, \alpha_{g}, \alpha_{b}$, are the contrast due to red, green, and blue color constituents, respectively, and $\beta_{r}, \beta_{g}, \beta_{b}$ denote the amount of brightness needed to be increased to or decreased from the respective red, green, and blue constituents $\mathbf{C}_{\boldsymbol{r}}, \mathbf{C}_{g}$, $\mathbf{C}_{\boldsymbol{b}}$ of the original color image $\mathbf{C}$ to the new color image $\mathbf{f}$. The outcome of the RMS contrast equalization technique different images is shown in Fig. 1.
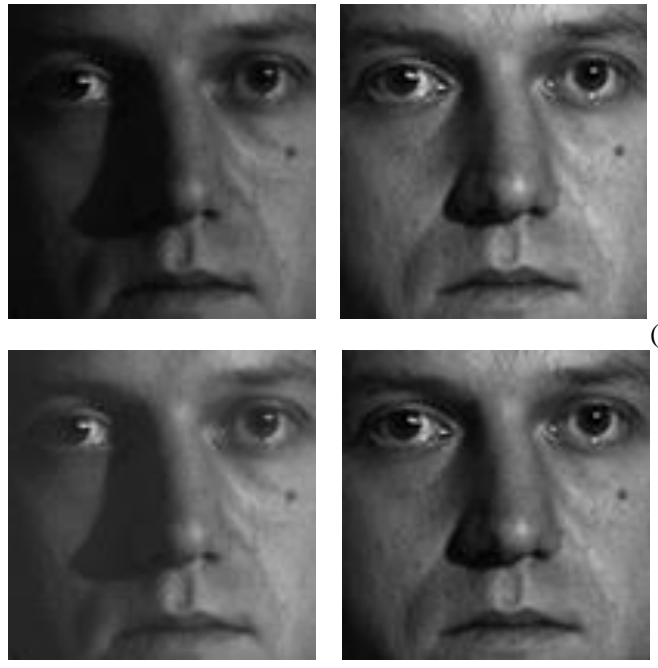

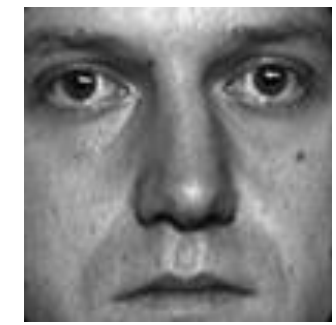

(a)Images with different brightness and contrast

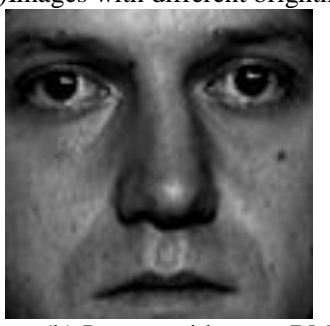

(b) Images with same RMS contras
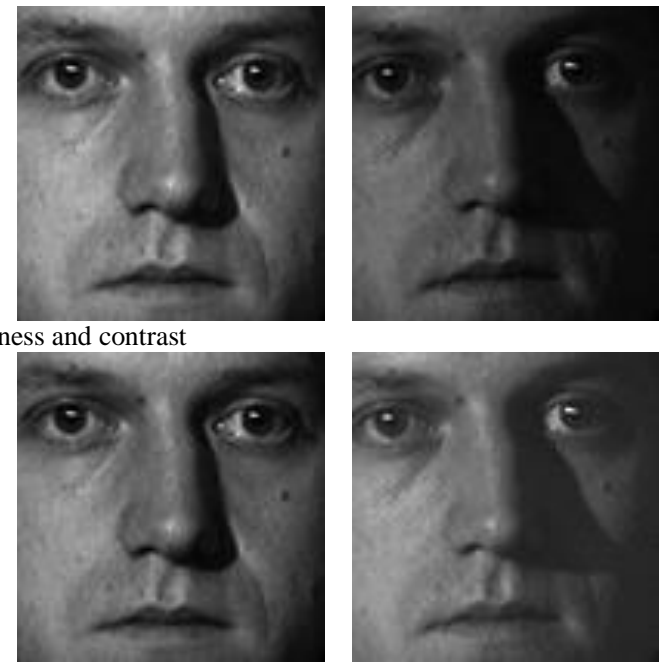

Fig. 1. RMS contrast equalization. Images were captured at illumination angles of $-38.4^{\circ},-21.6^{\circ},-0.2^{\circ}, 20.6^{\circ}, 37^{\circ}$, respectively, all images at a pose of $0^{\circ}$

\section{PRINCIPAL COMPONENT ANALYSIS}

Facial features are extracted employing principal components which are significant for face perception. This method is commenced with the computation of eigenvectors from the initial set of face images. New facial images are projected into the space stretched by eigenfaces and represented by weighted sum of the eigenfaces. These weights are applied to recognize the faces. The objective of the PCA is to yield the complete discrepancy on the training set of images and to describe this variation with a few variables. When the dimension of training data is increased, dimension of space complexity becomes a vital issue. The space complexity is extremely redundant when it represents faces because each pixel in a face is greatly correlated to other pixels.

PCA, a nonparametric statistical method concerned with explaining the covariance structure of a set of variables, is used to highlight the variation and convey strong patterns in a dataset. It allows us to explore, identify, and visualize the principal directions in which the data varies and representing the data to focus their resemblances and divergences. The principal reason behind using PCA is to decrease the dimension of space complexity. The maximum number of principal components is the number of variables in the original space [18]. The linear transformation maps the original $n$ dimensional space into an $m$-dimensional feature subspace. To reduce the dimension, some principal components can be discarded. The eigenfaces are the principal components of the original face images, achieved by the decomposition of PCA and eigenfaces are constructed employing eigenvectors [19].

\section{A. Eigenvectors}

An eigenvector, the representative vector of a square matrix, is a vector that does not change its direction under the associated linear transformation. Let $\mathbf{v}$ be a vector $(\mathbf{v} \neq 0)$, then it is an eigenvector of a square matrix $\mathbf{A}$, if $\mathbf{A v}$ is a scalar multiple of $\mathbf{v}$.

\section{B. Eigenfaces}

Eigenfaces are the set of eigenvectors which are used for human face recognition. These are represented as the eigenvectors which specify one of the dimensions of face image space. Eigenfaces provide significant characteristics that express the deviation in the group of face images. Each eigenvectors belongs to an eigenvalue associated with it and the eigenvectors having greater eigenvalues deliver more information on the face variation than the ones with lesser eigenvalues. Any new face image can be characterized as linear arrangement of these eigenfaces.

\section{FACE DETECTION}

A number of methods have been proposed for face detection, such as facial features extraction, knowledge-based approach, template matching, and color segmentation. This paper has combined template matching, skin color 
segmentation, and feature invariant approaches for face detection.

Skin color segmentation is established on the visual information of the human skin colors extracted from the image sequences. The human skin color differs from person to person and of different races, that is, chrominance and luminance components are different for different persons even in the same illumination environments. This research employs HSV color model for skin color segmentation.

In the HSV color space, a color is designated by three characteristics: hue, saturation, and value. Hue is the feature of visual impression that relates to color sensitivity linked with the prevailing colors, saturation infers the relative purity of the color component and value indicates the brightness of a color. The conversion from RGB space to HSV space is expressed by the equations [20-22]:

$$
\begin{gathered}
H=\left\{\begin{array}{l}
\operatorname{arc} \cos \frac{(R-G)+(R-B)}{2 \sqrt{(R-G)^{2}+(R-B)(G-B)}}, \quad B \leq G \\
2 \pi-\arccos \frac{(R-G)+(R-B)}{2 \sqrt{(R-G)^{2}+(R-B)(G-B)}}, B>G
\end{array}\right. \\
S=\frac{\max (R, G, B)-\min (R, G, B)}{\max (R, G, B)}, \\
V=\frac{\max (R, G, B)}{255},
\end{gathered}
$$

where $R, G, B$ are the red, green, and blue constituent values which exist in the range $[0,255]$.

Facial images are thresholded employing the hue histogram of the respective image. In this research, the hue values are chosen $h=[0,40]$. The detection of face area by such a hue segmentation process is illustrated in Figure 2.

To locate the face, an image pyramid is built from a set of facial images with different scales and resolutions. For this, a face template is moved from left to right and up to bottom over each image in the pyramid and calculate the matching probability at each position of the image segment under the template using minimum Manhattan distance. If the similarity value is greater than some threshold value, the existence of a face at that position and resolution is expected. From that position and resolution, the position and size of the face in the original image is being evaluated.

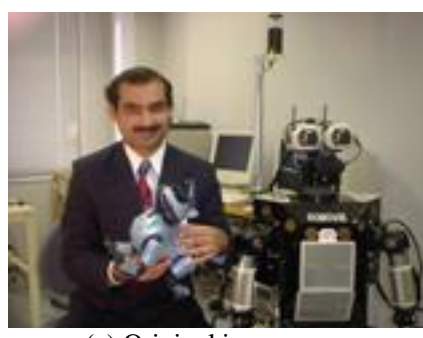

(a) Original image

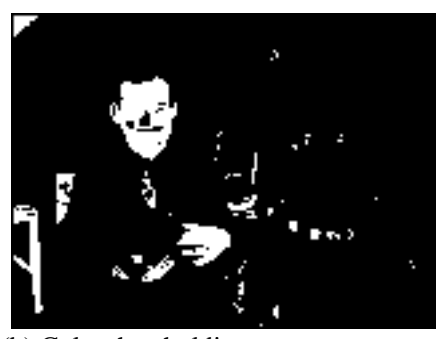

(b) Color thresholding
Fig. 2. Skin color segmentation

\section{FACE IMAGE NORMALIZAION}

The facial images are being normalized for face recognition. The original images are ostensibly the color images. These are transformed into gray scale images. The conversion from color image to grayscale image is given by the following equation:

$$
G_{n}=\frac{R_{n}+G_{n}+B_{n}}{3}
$$

where $R_{n}, G_{n}, B_{n}$ denote the red, green, and blue color constituents of the $n^{t h}$ pixel of the color image and $G_{n}$ is the gray level value of $n^{\text {th }}$ pixel of the gray scale image, and the resolution of the image is $M \times N$. The gray scale image is then scaled to $120 \times 120$ pixel using Eq. 8 .

$$
N\left(x_{m}, y_{m}\right)=M\left(\frac{x_{n}}{120} x_{m}, \frac{y_{n}}{120} y_{m}\right)
$$

where the coordinate of $n^{\text {th }}$ pixel of original gray scale image, $M\left(x_{n}, y_{n}\right)$ is converted into the $m^{t h}$ pixel of the scaled image and $N\left(x_{m}, y_{m}\right)$ is the coordinate of that pixel.

\section{FACE RECOGNITION}

This research considers the face recognition system dividing into two parts: initialization and recognition. In the initialization phase, the system is learned by creating eigenvectors of a training set of face images. The fundamental procedure employed for face recognition process is shown in Figure 3.

Algorithm 1 is applied for initialization of face recognition system.

\section{Algorithm 1 (Initialization)}

Input: A set of facial images known as Training set $\left(\Gamma_{k}\right)$.

Output: Form feature vectors for each image.

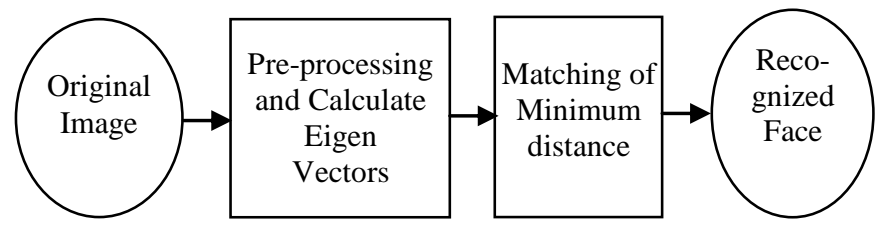

Fig. 3. Basic Steps used for Face Recognition

Method: The feature vector is constructed in the following steps.

1. Calculate mean matrix $\Psi$. Then subtract this mean from the original faces $\left(\Gamma_{k}\right)$ to calculate the feature vector $\left(\varphi_{k}\right)$, where

$$
\psi=\frac{1}{N} \sum_{k=1}^{N} \Gamma_{k},
$$

2. Find the covariance matrix $\mathbf{c}$ as follows: 


$$
\mathbf{c}=\frac{1}{N} \sum_{n=1}^{N}\left(\Gamma_{k}-\psi\right)\left(\Gamma_{k}-\psi\right)^{T}
$$

3. Compute the eigenvectors and eigenvalues of $\mathbf{c}$.
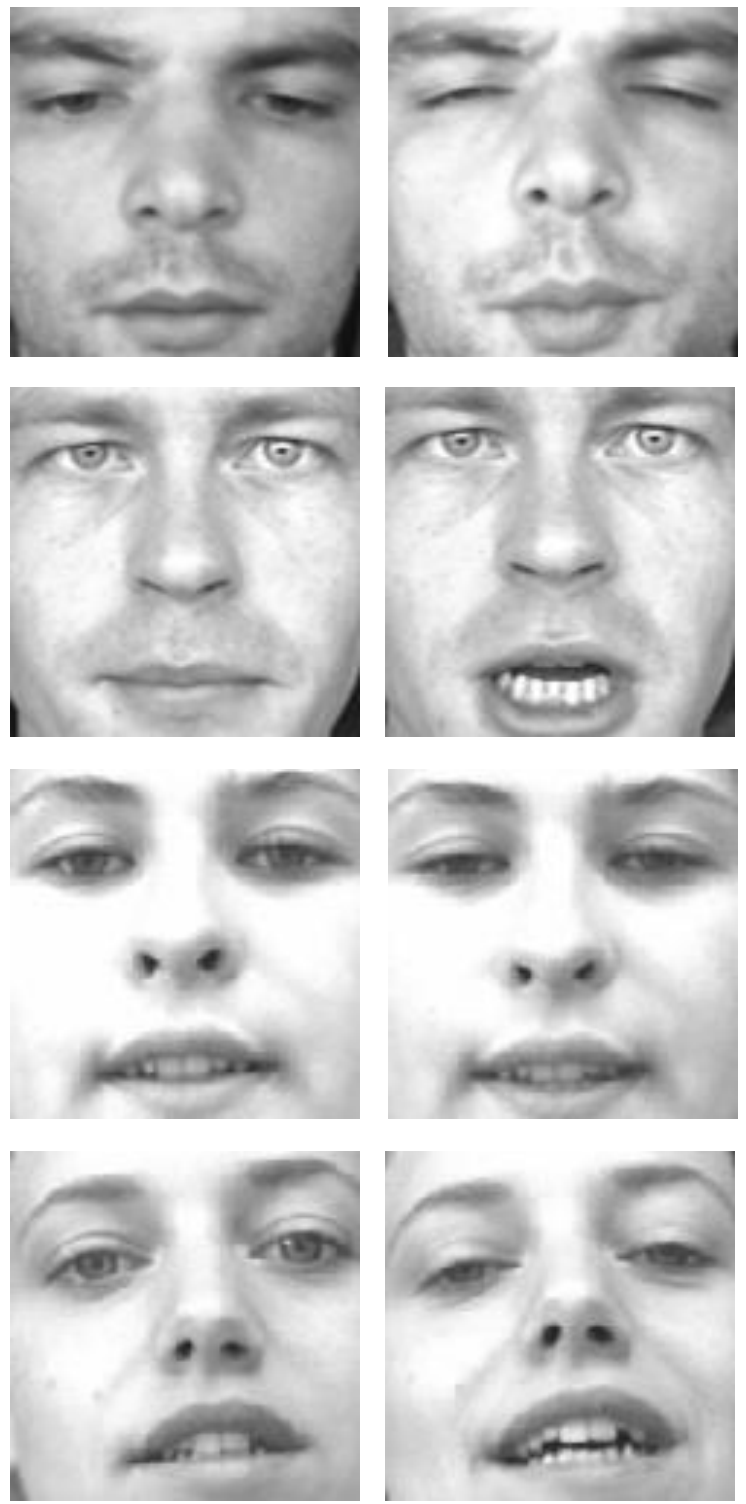

4. The $N$ significant eigenvectors are selected on the basis of biggest equivalent eigenvalues.

5. Project all the face images into these eigenvectors and form the feature vectors of each face image.
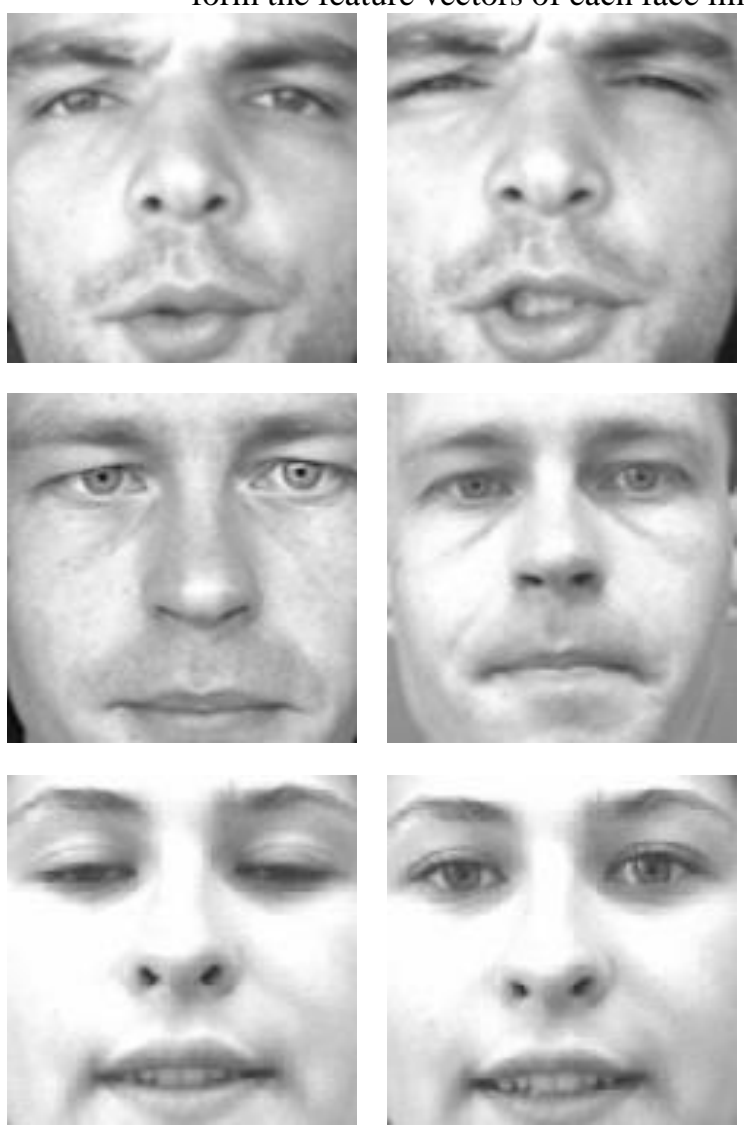

Fig. 4. Training face images
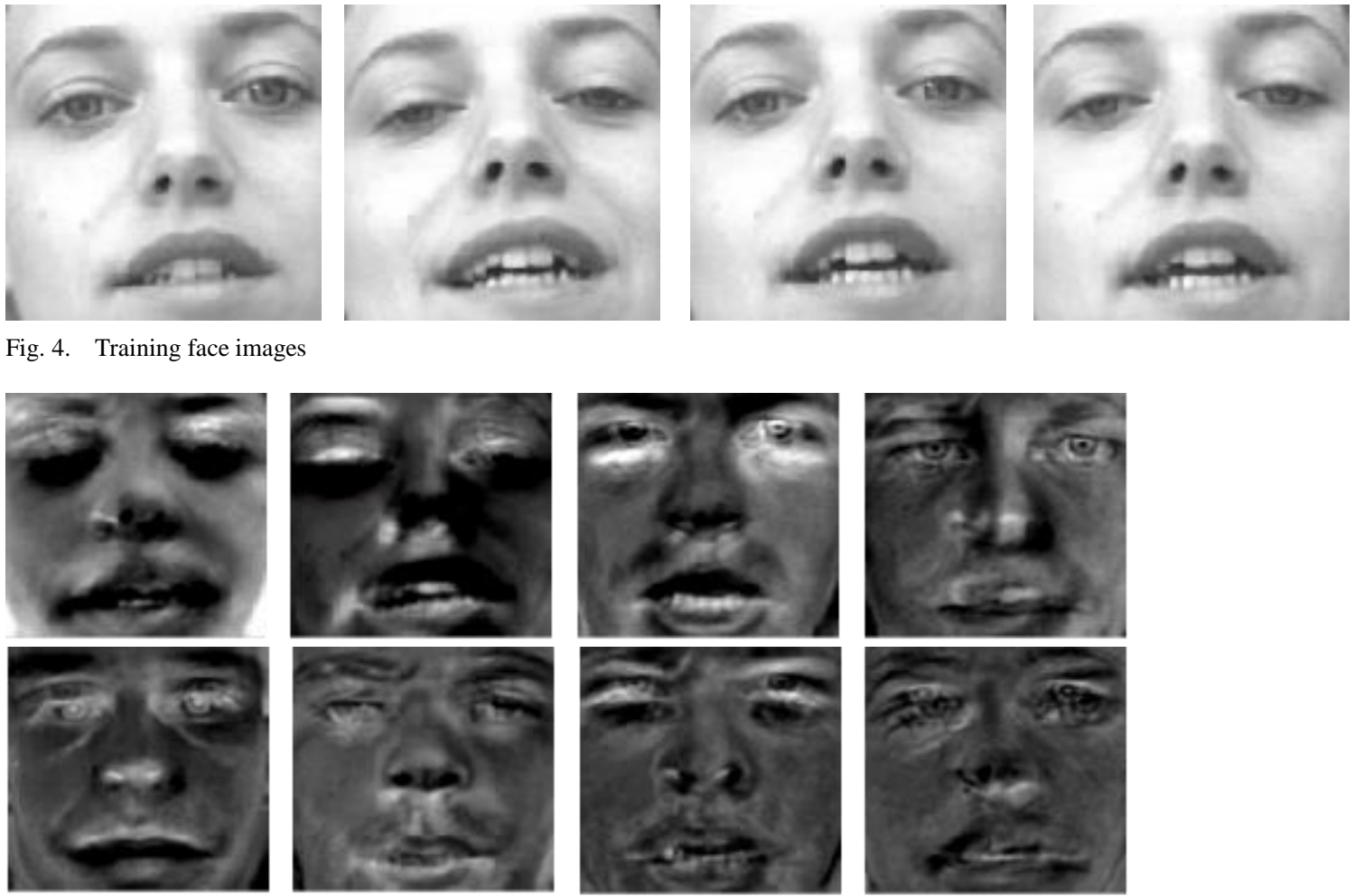

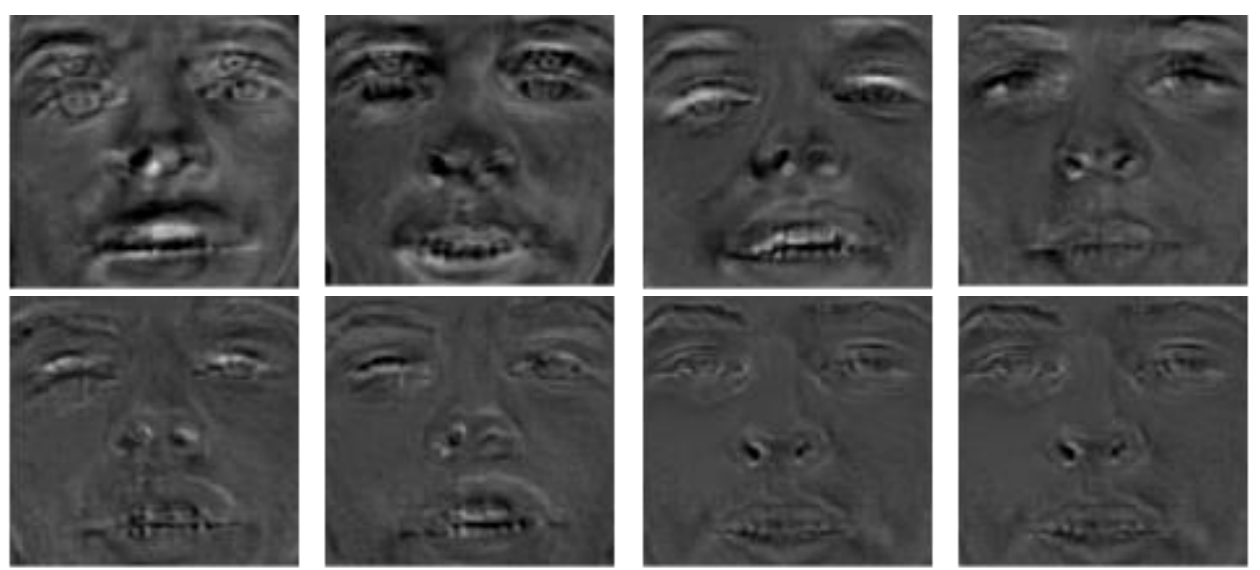

Fig. 5. Eigenfaces with highest eigen values

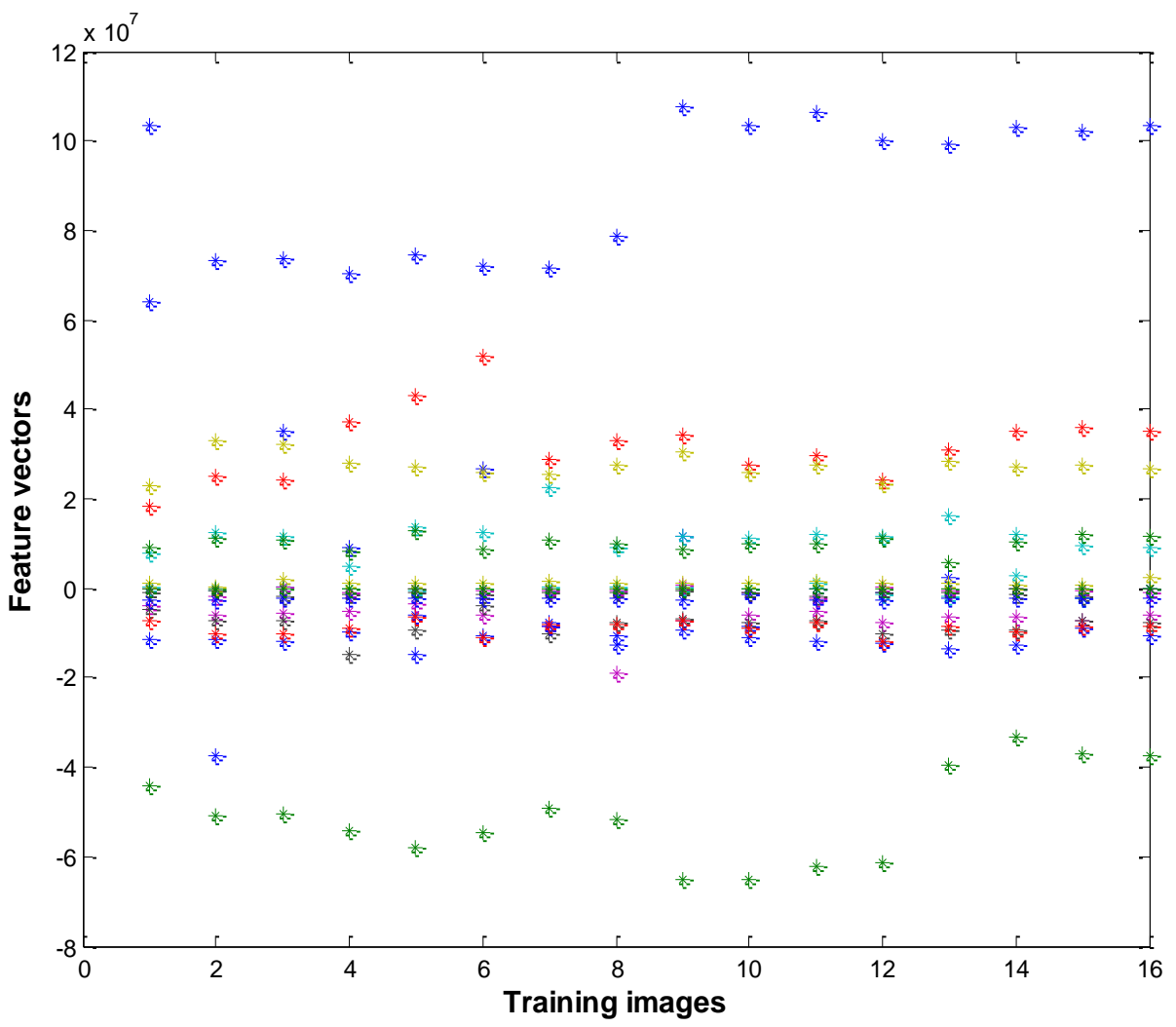

Fig. 6. Distribution of the feature vectors for a set of facial images

After getting the feature vectors, Algorithm 2 is employed to recognize an unknown face.

\section{Algorithm 2 (Recognition)}

Input: An unknown image $\boldsymbol{I}$.

Output: Recognize the image $\boldsymbol{I}$.

Method: The FP-tree is constructed in the following steps.

1. For a given input image $\boldsymbol{I}$, compute a set of vectors based on $N$ eigenfaces by projecting the new image onto each of eigenfaces [2].
2. Compute the difference between the projected vector and each face image feature vector.

3. Classify the weight pattern as either known or unknown person.

4. The weight pattern can be compared with known weight patterns to match faces [2].

This analysis drastically reduces the order of the number of pixels in the images $\left(N^{2}\right)$, to the order of the number of images in the training set $\left(\Gamma_{i}\right)$. 
A few sample face images and the corresponding eigenfaces are shown in Figures 4 and 5, respectively. Each eigenface departs from the original grayscale image where some facial features diverge among the set of training faces. Thus, eigenfaces can be regarded as a more or less mapping of the disparities between faces.

\section{EXPERIMENTAL RESULTS}

In order to justify the effectiveness of the algorithm several experiments were carried out with a training set of 500 images of 100 persons both male and female from CMU database [23] and University of Essex Computer Vision Science Research Projects dataset [24]. All images were in RGB color level which were normalized to gray level with dimension of $120 \times 120$. There were 70 subjects in the training set. Each subject had 5 images with frontal view with $\pm 5^{\circ}$ different poses (like left, right, up, and down). The training arrangement is summarized in Table 1.

TABLE I. TRAINING ARRANGEMENT FOR IMAGES

\begin{tabular}{|l|l|}
\hline $\begin{array}{l}\text { No. of images taken for the } \\
\text { training procedure }\end{array}$ & $50 \times 5+50 \times 5=500$ \\
\hline Size & $120 \times 120$ \\
\hline Format & BMP and PGM \\
\hline Output & $\begin{array}{l}\text { Normalized images of the face } \\
\text { images and Eigenfaces }\end{array}$ \\
\hline
\end{tabular}

In this research, the eigenvectors of covariance matrix were computed by aggregating all deviations of training images from the average image. Since the training set contains 100 individuals, 100 eigenvectors have been used to represent the training set. The distribution of the feature vectors for a set of facial images is shown in Figure 6. Later on, images are being categorized in different lighting conditions. The overall results under different lighting conditions is shown in Table 2.

The performance of the proposed method has been compared to other similar methods which have been used the same experimental methodology. Table III shows the comparison of the performances between the proposed method (PCA+RMS contrast scaling) and the methods, as reported by [15] and [19].

TABLE II. TRAINING ARRANGEMENT FOR IMAGES

\begin{tabular}{|l|l|l|}
\hline $\begin{array}{l}\text { Lighting } \\
\text { conditions }\end{array}$ & $\begin{array}{l}\text { Total number of images } \\
\text { taken for the test }\end{array}$ & $\begin{array}{l}\text { Correctly recognized } \\
\text { (Success rate) }\end{array}$ \\
\hline Bright & 350 & $97.5 \%$ \\
\hline Dark & 50 & $82 \%$ \\
\hline Foggy & 100 & $86 \%$ \\
\hline
\end{tabular}

TABLE III. COMPARISON OF THE PERFORMANCES BETWEEN THE PROPOSED METHOD AND OHHER SIMILAR METHODS

\begin{tabular}{|l|l|l|}
\hline Methods & $\begin{array}{l}\text { Total number of images } \\
\text { taken for the test }\end{array}$ & Average Success rate \\
\hline PCA+RBF & 400 & $87.25 \%$ \\
\hline PCA & 350 & $80.86 \%$ \\
\hline $\begin{array}{l}\text { PCA+RMS } \\
\text { contrast scaling }\end{array}$ & 100 & $93.6 \%$ \\
\hline
\end{tabular}

\section{CONCLUSION}

This article presents an eigenface based approach for face recognition, where the eigenvectors of the covariance matrix of a set of representative images are explored. Recognition is accomplished by computing the distance between characteristic vectors from the eigenvectors space. This eigenface method is robust for face recognition which works fine under controlled environment. But the main limitation of this approach is contained in the management of face images with diversive facial expressions, lighting conditions and wearing glasses. Lighting problems are overcomed by RMS contrast stretching. The face database in this work contains 500 face images and the proposed method provides satisfactory result. It can recognize both the known and unknown images in the database in various conditions with accuracy more than $80 \%$. Our next target is to extend the eigenface approach for live video stream so that any person can be identified at his or her own workplace.

\section{REFERENCES}

[1] L. Sirovich and M. Kirby, "Low-dimensional procedure for the characterization of human faces". Journal of Optical Society of America, 4(3):519-524 (1987).

[2] M. Turk and A. Pentland, "Eigenfaces for recognition", Journal of Cognitive Neuroscience, 3(1):71-86 (1991).

[3] L. Wiskott, J.M. Fellous, N. Kruger, CV Malsburg, "Face recognition by elastic bunch graph matching", IEEE Transaction on Pattern Analysis and Machine Intelligence, 19(7):775-779 (1997).

[4] M. Lades, J.C. Vorbruggen, J. Buhmann, J. Lange, C.V. Malsburg, C. Wurtz, W. Konen, "Distortion invariant object recognition in the dynamic link architecture, "IEEE Transaction on Computers", 42(3):300-311 (1993).

[5] S. Shan, P. Yang, X. Chen, W. Gao, "AdaBoost gabor fisher classifier for face recognition", Proceedings of IEEE International Workshop on Analysis and Modeling of Faces and Gestures, 278-291 (2005).

[6] B. Zhang, S. Shan, X. Chen, W. Gao, "Histogram of gabor phase patterns: a novel object representation approach for face recognition", IEEE Transection on Image Processing, 16(1):57-68 (2007).

[7] C. Liu and K. Wechsler, "Gabor feature based classification using the enhanced fisher linear discriminate model for face recognition", IEEE Transaction on Image Processing, 11(4):467-476 (2002).

[8] M. Kirby and L. Sirovich, "Application of the Karhunen-Loeve procedure for the characterization of human faces", IEEE Transaction on Pattern Analysis and Machine Intelligence. 12(1):103-108 (1990). 
[9] D. Terzopoulos, K. Waters, "Analysis of facial images using physical and anatomical models", Proceedings of $3^{\text {rd }}$ International Conference on Computer Vision, 727-732 (1990).

[10] C.J. Wu and J.S. Huang, "Human face profile recognition by computer". Pattern Recognition, 23(1):255-259 (1990).

[11] B.S. Manjunath, R. Chellappa, and C. Malsburg, "A feature based approach to face recognition", Transection of IEEE, 373-378 (1992).

[12] M.A. Kerin, T.J. Stonham, "Face recognition using a digital neural network with self-organizing capabilities", Proceedings of $10^{\text {th }}$ International Conference on Pattern Recognition, 738-741 (1990).

[13] O. Nakamura, S. Mathur, T. Minami, "Identification of human faces based on isodensity maps", Pattern Recognition, 24(3):263-272 (1991).

[14] A.L. Yuille, D.S. Cohen, P.W. Hallinan, "Feature extraction from faces using deformable templates", Proceedings of CVPR, 104-109 (1989).

[15] E. Peli E, "Contrast in complex images", Journal of Optical Society, 7(10): 2032-2040 (1990).

[16] S. Thakur, J.K. Sing, D.K. Basu, M. Nasipuri, " Face recognition using principal component analysis and RBF neural networks", IJSSST, 10(5): 7-15 (2012).

[17] M.A. Bhuiyan, F.A. Alsaade, "Genetic search for face detection", Proceedings of the World Congress on Engineering, I:157-162 (2015).
[18] M. Murali, "Principal component analysis based feature vector extraction", Indian Journal of Science and Technology. 8(35):1-4 (2015).

[19] D. Chakraborty, S.K. Saha, M.A. Bhuiyan, "Face recognition using eigenvector and principle component analysis", International Journal of Computer Applications, 50(10):42-49 (2015).

[20] M.A. Bhuiyan, "Content-based image retrieval for image indexing", International Journal of Advanced Computer Science and Applications, 6(6):71-79 (2015).

[21] D. Androutsos, K.N. Plataniotis, A.N. Venetsanopoulos, "A model vector-based approach to color image retrieval using a vector angularbased distance measure", Computer Vision and Image Understanding, 75(1):46-57 (1999).

[22] M.A. Bhuiyan, A. Vuthichai, S. Muto, H. Ueno, "On tracking of eye for human-robot interface", International Journal of Robotices and Automation, 19(1):42-54 (2004).

[23] T. Sim, S. Baker, M. Bsat, "The CMU Pose Illumination and Expression (PIE) Database", Proceedings of the 5th International Conference on Automatic Face and Gesture Recognition, 2002.

[24] M.A. Bhuiyan, C.H. Liu, "Intelliget vision system for human-robot interface", Proceedings of World Academy of Science, Engineering and Technology, 28:57-63 (2007). 